\title{
Acute effect of strength training on mood of patients with fibromyalgia syndrome
}

\author{
A. Andrade, R. de Azevedo Klumb Steffens, S. Mendes Sieczkowska, \\ D. Reis Coimbra, G. Torres Vilarino \\ Health and Sports Science Center, Santa Catarina State University, Brazil
}

\begin{abstract}
SUMMARY
Fibromyalgia syndrome (FM) is a musculoskeletal disorder characterized by chronic pain and frequently associated changes in mood states. The aim of this study was to analyze the acute effect of strength training (ST) sessions on the mood states of patients with fibromyalgia. A total of 110 FM patients were eligible for this study. After the inclusion criteria, twenty-eight women with FM (mean age: 51.88 \pm 10.22 years) performed three sessions of ST. Each training session worked the main muscle groups and lasted 60 min. Three sets of 12 repetitions were performed with 1-min intervals between them. Outcome measures were assessed at baseline, after one session, and after three ST sessions. The Brunel Mood Scale (BRUMS) was used to assess mood states, and the Wilcoxon test was used to verify differences in mood after one and three ST sessions. The ST practice had positive effects on the patients' mood states after a single session. Reductions in anger, mental confusion, mood depression, fatigue, and tension were observed. The results of the $3^{\text {rd }}$ ST session were similar. We concluded that a single ST session was sufficient to improve the mood states of patients with fibromyalgia.
\end{abstract}

Key words: Fibromyalgia; exercise; resistance training mood; affective disorder.

Reumatismo, 2019; 71 (3): 141-147

\section{INTRODUCTION}

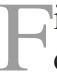
ibromyalgia syndrome (FM) is a musculoskeletal disease that combines generalized pain for a period of more than three months in four out of five regions of the body (quadrants and axial skeleton) (1). In addition to pain, other common symptoms are changes in mood, anxiety, depression, sleep disorders, cognitive problems, and fatigue (2-5).

It has been noted that FM patients who experience mood changes have similar psychological characteristics, presenting feelings of vulnerability and helplessness (5). Some studies have shown that these patients have high rates of psychological disorders, especially depression and excessive anxiety (6). Interventions to improve the health of patients with FM should therefore consider mood changes, alongside physical symptoms such as pain and fatigue. Mood reflects a complex pattern of behaviors, feelings, thoughts, and physical and emotional states; a mood consists of six factors: anger, confusion, mood depression, fatigue, vigor, and tension (7-10).

To improve the physical and psychological symptoms and consequently the general health of patients with FM, physical exercise has been recommended as an alternative treatment in recent years (11-15). Among various types of physical exercise, strength training (ST) has been shown to be an effective treatment option in helping to reduce the most common symptoms of the syndrome (16-20). However, few studies have investigated the effects of ST on particular psychological variables (14). In a meta-analysis conducted by Busch et al. (14), only three studies evaluated the effects of ST on psychological variables. It is important to analyze the acute effects of physical exercise on patients with several pathologies; some studies have already presented positive results (21-23). A study carried out by Herring et al. (24) has found that exercise significantly improves anxiety, energy levels, and fatigue.

When considering the acute effects of ST, the gap is even greater; to date, no stud- $\overline{\text { Corresponding author: }}$ Alexandro Andrade Health and Sports Science Center Santa Catarina State University,

Pascoal Simone 358 - Coqueiros CEP 88080-350, Brazil

E-mail: alexandro.andrade.phd@gmail.com 


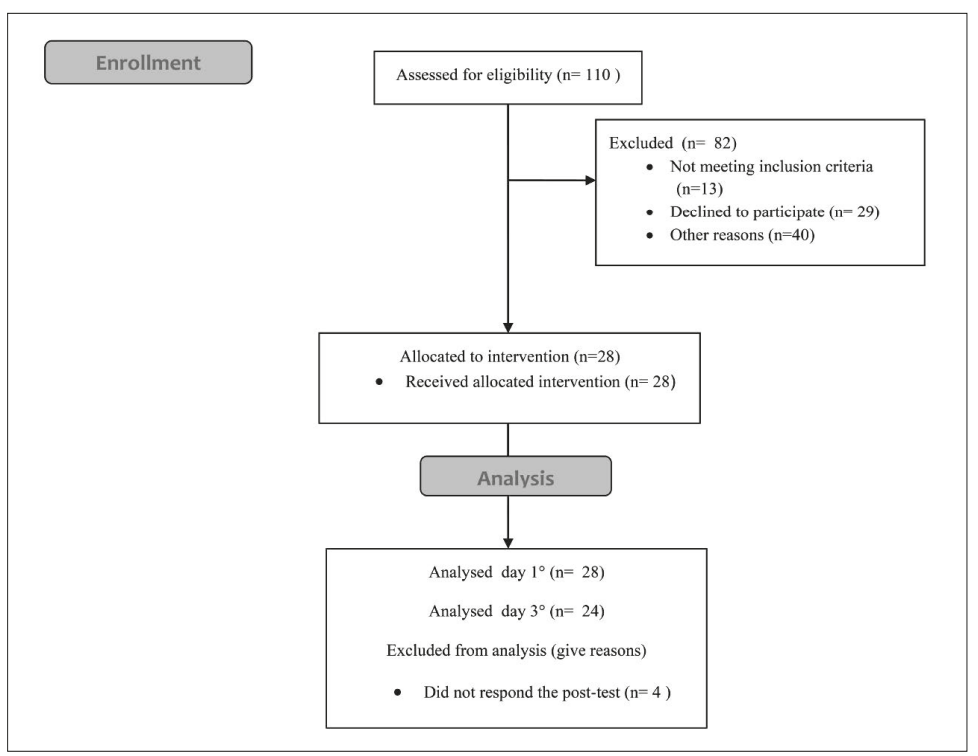

Figure 1 - The flowchart of eligibility of study participants.

ies have evaluated the acute effects of ST on the psychological variables of patients with FM. Investigating treatment alternatives that have the potential to reduce psychological symptoms quickly is fundamental to improving the quality of life of this population. The aim of the present study is therefore to analyze the acute effect of ST on the mood states of patients with FM, after one and three strength training sessions.

\section{MATERIALS AND METHODS}

\section{Design}

This has been a clinical trial carried out among patients with FM. It was conducted in accordance with the ethical standards required by the Declaration of Helsinki and Resolution 466/12 of the National Health Council of Brazil; it was approved by the Research Ethics Committee Involving Humans, Santa Catarina State University (UDESC): protocol no. 24584213.0.0000.0118; opinion No 706,588 . The present study has been registered in the Brazilian registry of clinical trials under the number RBR-74pcmw.

A total of $110 \mathrm{FM}$ patients were eligible to participate in the study, having met the following inclusion criteria:
1) a medical diagnosis of FM, based on the American College of Rheumatology $(1,25)$;

2) female gender;

3) age greater than 18 years;

4) no physical exercise in the last three months. Patients were recruited through newspaper advertisements and pamphlets distributed in hospitals, as well as health postings in the cities of Florianópolis and São José, SC, Brazil.

Patients interested in participating in the study were contacted by telephone. During an initial evaluation, researchers explained the objectives, evaluation procedures, and relevance of the study. Patients were guaranteed complete confidentiality; those who chose to participate completed written consent forms. Reasons for exclusion: patients with others severe health conditions, who performed physical exercise, did not want to participate in the acute protocol and those who did not have time for the study activities were excluded (4). At the end of the selection process, 28 patients with FM were analyzed (Figure 1).

\section{Measurements}

The data were obtained via two instruments: a self-reported instrument was used to obtain sociodemographic and clinical data; the Brunel Mood Scale (BRUMS) was used to verify mood states $(5,26)$.

\section{Demographic and clinical characteristics}

The study questionnaire gathered information about the participants' sociodemographic and clinical characteristics, including age, marital status, occupation, time of diagnosis, most common symptoms, and medical diagnosis of depression.

\section{Brunel Mood Scale (BRUMS)}

The Brunel Mood Scale (BRUMS) (5, 26) was used to evaluate mood states. The questionnaire consisted of 24 questions and participants responded to each question by selecting a numerical response between zero (not at all) and four (extremely). The BRUMS was applied four times: i) immediately before the first session of strength training; ii) after the first session; iii) be- 
fore the third session; and iv) after the third session.

\section{Intervention}

Patients took part in strength training three times a week at Santa Catarina State University (UDESC), with a 48-h interval between sessions. Each session lasted 60 minutes. All exercises were overseen by experienced physical education teachers and physiotherapists. A training session consisted of a 10-minute warm-up, followed by 50 minutes of resistance exercises for large and small muscle groups. Each participant had an individualized ST program, prescribed within a safe and healthy range. The weights used in each exercise were assessed subjectively by the participants, who were instructed to aim for slight discomfort in the final repetitions. The patients performed three sets of 12 repetitions, with a 1-minute interval between each series. The main part of each session consisted of exercises targeting major muscle groups, including the muscles of the chest, the latissimus dorsi, biceps, triceps, quadriceps, hamstrings, shoulders, and calves. Patients performed the following exercises: knee extension, knee flexion, bench press, fly, adductors, low rowing, high pulley, elbow extension, lateral raise, arm curl, standing calf raise, and abdominal crunch.

\section{Statistical analysis}

Data were analyzed using descriptive statistics (mean, standard deviation) and inferential statistics. A Shapiro-Wilk test verified the normality of the data. When the data were distributed normally, a paired Student $t$ test was used. A Wilcoxon test was used to analyze non-parametric data. The significance level of all tests of hypotheses was $5 \%(\mathrm{p}<0.05)$. In addition, to assess the difference between pre-ST and post-ST results, the effect size (r) was calculated using equations $r=Z / \sqrt{ } N$ for non-parametric data and Hedges $g=M_{1}-M_{2} / \mathrm{SD}^{*}{ }_{\text {pooled }}$ ), interpreted as follows: $r<0.30$ (small); $r>0.30$ and $<0.50$ (medium); and $r>0.50$ (large). To represent the acute effect of the ST session on mood, the delta $(\Delta)$ and $\Delta \%$ were calculated, based on the difference between the mean post-intervention mood and the pre-intervention mood divided by the mean pre-intervention mood multiplied by $100\left[\Delta \%=\left(\left(\hat{x} \operatorname{Mood}_{p o ́ s}-\not{x} \operatorname{Mood}_{p r e ́}\right)\right)\right.$ $\left.\left.x \operatorname{Mood}_{\text {pré }}\right) * 100\right]$.

The sample size calculations used to detect a statistical difference for a single-tailed hypothesis test, based on an effect size of 0.5 , involved a $1-\beta$ error probability of 0.8 and a $\mathrm{p}<0.05$ for the dependent variable. The data were analyzed using $G^{*}$ Power (27) and SPSS 20.0 software (IBM, U.S.).

\section{RESULTS}

The study included 28 women with FM; their mean age was $51.88 \pm 10.22$. Participants had an average time of diagnosis of $7.75 \pm 7.70$ years, were married $(64.3 \%)$, had not finished elementary school (28.6\%), were currently working (78.6\%) and most patients had a medical diagnosis of depression $(85.7 \%)$. The main symptoms they reported were generalized pain $(89.3 \%)$, tiredness $(85.7 \%)$, non-restorative sleep $(71.4 \%)$, joint stiffness $(67.9 \%)$, and fatigue $(71.4 \%)$. The data are shown in Table I.

ST had a positive effect on the patients' mood states (Table II). After a single ses-

Table I - Sociodemographic and clinical profile of fibromyalgia patients participating in the study.

\begin{tabular}{|c|c|}
\hline & $\begin{array}{c}\text { Group ST } \\
(\mathrm{n}=28)(\dot{x} \pm d p)\end{array}$ \\
\hline Age, years & $51.9 \pm 10.22$ \\
\hline Diagnostic time & $7.8 \pm 7.7$ \\
\hline $\begin{array}{l}\text { Marital status } \\
\text { With partner } \\
\text { Without partner }\end{array}$ & $\begin{array}{l}18(64.3) \\
10(35.7)\end{array}$ \\
\hline $\begin{array}{l}\text { Work } \\
\text { Yes } \\
\text { No } \\
\#\end{array}$ & $\begin{array}{c}22(78.6) \\
5(17.9) \\
1(3.6) \\
\end{array}$ \\
\hline $\begin{array}{l}\text { Diagnosis of depression } \\
\text { Yes } \\
\text { No }\end{array}$ & $\begin{array}{c}24(85.7) \\
4(14.3)\end{array}$ \\
\hline $\begin{array}{l}\text { Most common symptoms } \\
\text { Generalized pain } \\
\text { Tiredness } \\
\text { Sleep disturbances } \\
\text { Stiffness } \\
\text { Fatigue }\end{array}$ & $\begin{array}{l}25(89.3) \\
24(85.7) \\
20(71.4) \\
19(67.9) \\
20(71.4)\end{array}$ \\
\hline
\end{tabular}

\# missing data. 
Table II - Effect of ST practice on the mood state after the first session and after three practice sessions.

\begin{tabular}{|l|c|c|c|c|c|c|}
\hline \multirow{2}{*}{ Mood } & \multicolumn{3}{|c|}{ Assessment $1^{\text {st }}$ session $(\mathbf{n}=28)$} & \multicolumn{3}{c|}{ Assessment $3^{\text {rd }}$ session $(\mathbf{n}=24)$} \\
\cline { 2 - 7 } & Before $(\dot{x} \pm d \mathbf{d p})$ & After $(\dot{x} \pm d p)$ & $\mathbf{p}$ & Before $(\dot{x} \pm d p)$ & After $(\dot{x} \pm d p)$ & $\mathbf{p}$ \\
\hline Anger & $3.46 \pm 5.17$ & $1.04 \pm 1.95$ & $0.017^{*}$ & $3.2 \pm 5.0$ & $1.0 \pm 2.1$ & 0.05 \\
\hline Mental confusion & $3.57 \pm 4.19$ & $2.11 \pm 2.92$ & $0.004^{*}$ & $3.4 \pm 4.1$ & $2.0 \pm 2.9$ & $0.005^{*}$ \\
\hline Mood Depression & $4.93 \pm 3.93$ & $1.82 \pm 2.20$ & $0.0001^{*}$ & $4.8 \pm 4.0$ & $1.9 \pm 2.3$ & $0.0001^{*}$ \\
\hline Fatigue & $7.29 \pm 4.78$ & $5.00 \pm 3.63$ & $0.018^{*}$ & $7.0 \pm 4.8$ & $4.8 \pm 3.7$ & 0.034 \\
\hline Tension & $6.32 \pm 3.92$ & $3.86 \pm 2.95$ & $0.001^{*}$ & $6.1 \pm 3.7$ & $4.0 \pm 3.0$ & $0.002^{*}$ \\
\hline Vigor & $6.89 \pm 2.66$ & $7.36 \pm 3.23$ & 0.404 & $6.5 \pm 2.3$ & $7.0 \pm 2.9$ & 0.231 \\
\hline
\end{tabular}

$x \pm d p$ : mean \pm standard deviation; *significant difference.

sion, reductions were observed in anger $(\mathrm{Z}=-2.394, \mathrm{p}=0.017 ; \quad r=0.45$, moderate), mental confusion $(\mathrm{Z}=-2.880, \mathrm{p}=0.004$; $r=0.54$, large $)$, mood depression $(\mathrm{Z}=-3.927$, $\mathrm{p}=0.000 ; r=0.74$, large $)$, fatigue $(\mathrm{t}=-2.521$, $\mathrm{p}=0.018 ; r=0.44$, moderate), and tension $(\mathrm{Z}=-3.367, \mathrm{p}=0.001 ; r=0.64$, large $)$. The only variable that did not undergo a significant change was vigor $(\mathrm{t}=0.847, \mathrm{p}=0.404)$.

The results of the $3^{\text {rd }}$ ST session show reductions in mental confusion $(\mathrm{Z}=-2.777$, $\mathrm{p}=0.005 ; r=0.57$, large), mood depression $(\mathrm{Z}=-3.519, \mathrm{p}=0.0001 ; r=0.72$, large $)$, fatigue $(\mathrm{Z}=-2.117, \mathrm{p}=0.034 ; r=0.43$, moderate), and tension $(\mathrm{Z}=-3.060, \mathrm{p}=0.034$; $r=0.63$, large). No change was observed in anger $(\mathrm{Z}=-1.962, \mathrm{p}=0.05)$ or vigor $(\mathrm{Z}=0.198, \mathrm{p}=0.231)$.

Figure 2 shows the effects of the first ST session on the mood of patients with FM.

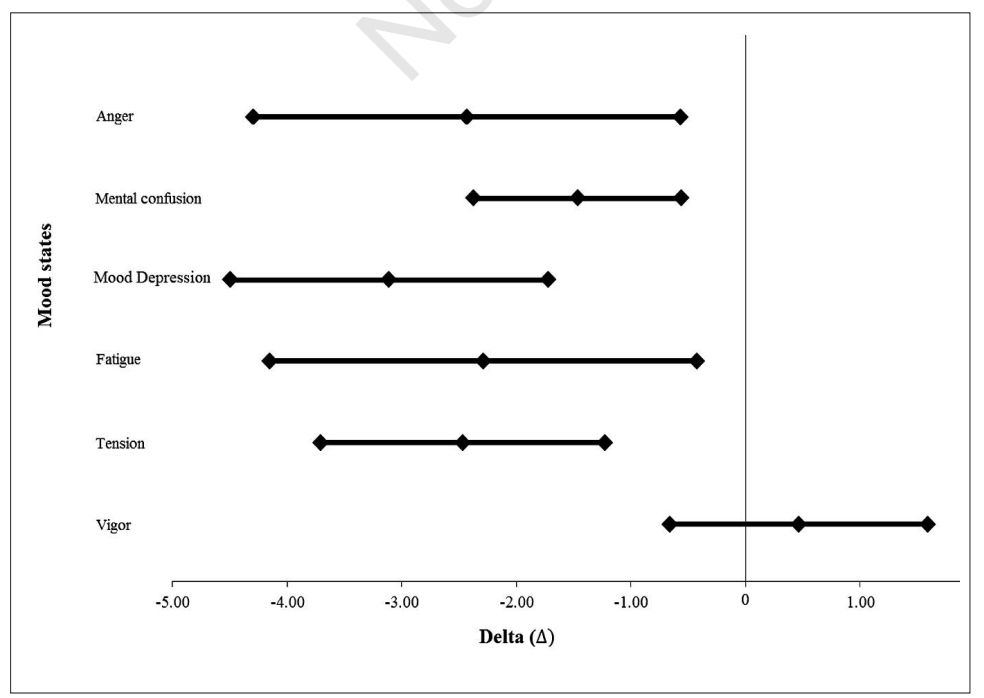

Figure 2 - Mood States after one session of Strength training.
A single ST session improved vigor in $7 \%$ of patients $(\Delta=0.46 ; 95 \% \mathrm{CI}=-0.66$ to 1.59$)$, reduced anger in median $70 \%(\Delta=-2.43$; $95 \% \mathrm{CI}=-4.29$ to -0.57$)$, mental confusion in $41 \%(\Delta=-1.46$; $95 \% \mathrm{CI}=-2.37$ to -0.56$)$, mood depression in $63 \%(\Delta=-3.11 ; 95 \%$ $\mathrm{CI}=-4.50$ to 1.72$)$, fatigue in $31 \%(\Delta=-2.29$; $95 \% \mathrm{CI}=-4.15$ to -0.43$)$, and tension in $39 \%(\Delta=-2.46 ; 95 \% \mathrm{CI}=-3.70$ to 1.23$)$.

After three sessions of ST, vigor improved in median $11 \%(\Delta=0.71 ; 95 \% \mathrm{CI}=-0.48$ to 1.90$)$, with reductions of anger in $78 \%$ $(\Delta=-2.46$; $95 \% \mathrm{CI}=-4.40$ to 0.51$)$, mental confusion in $47 \%(\Delta=-2.46 ; 95 \% \mathrm{CI}=$ -3.70 to 1.23 ), mood depression in $60 \%$ $(\Delta=-2.83 ; 95 \% \mathrm{CI}=-4.57$ to 1.10$)$, fatigue in $39 \%(\Delta=-2.75 ; 95 \% \mathrm{CI}=-4.68$ to 0.82$)$, and tension in $44 \%(\Delta=-2.67 ; 95 \% \mathrm{CI}=-$ 4.14 to 1.19$)$. Figure 3 shows the delta (\%) results of the effect of ST on the mood of FM patients after three practice sessions.

\section{DISCUSSION}

Our study analyzed the acute effect of one and three ST sessions on the mood states of patients with FM. The results demonstrate that an ST session improved the mood of these patients, significantly reducing anger, mental confusion, mood depression, fatigue, and tension. These results demonstrate acute positive responses to physical exercise, as have been found in patients with other diseases $(22,28)$.

The implications of the acute effect of physical exercise have been studied in relation to heart disease, diabetes, hyperlipemia, pulmonary diseases, psychological disorders, and other conditions. Previ- 
ous studies have shown beneficial changes to the patients' mental health (29-31), confirming that several exercise modalities can positively modify mood. Research has also indicated that the acute effects of exercise can reduce anxiety, as well as improve cognition and executive function in people undergoing an intervention $(21,22,32)$.

It is common for patients with chronic pain to have some sort of mood disorder (33). Patients with FM present rates of depression above $80 \%$, and anxiety over $32 \%$ $(3,34,35)$. A depressed mood may be a predictor for certain treatment responses (36); for these reasons, it is necessary to investigate short-term treatment strategies that may reduce these symptoms. Despite the urgency of this issue, few studies have explored the effects of physical exercise, either on mood, or on psychological variables in patients with FM. The meta-analysis developed by Busch et al. (14) includes only three studies that evaluated the effect of physical exercise on mood states; none of these evaluated the acute effect. In a study by Bircan et al. (37), patients with FM engaged in eight weeks of ST or aerobic exercise, revealing the effect of exercise on depression, anxiety, and mental health. At the end of the study, mood depression was reduced in both groups, with no significant difference between them. However, anxiety and mental health issues were not mitigated, following the intervention. After 15 weeks of ST, Ericsson et al. (20) did not find significant differences in the levels of depression and anxiety in patients with FM. Despite these conflicting results, depression tends to decline after ST practice. The results of the present study demonstrate that a mood depression is reduced after a single session of ST, as well as after three sessions. Andrade et al. (3) analyzed 215 patients with FM and found that those who exercised regularly had fewer depressive symptoms; Häkkinen et al. (38) found a reduction in depression after 21 weeks of ST.

No previous study has evaluated the acute effect of TF on the psychological symptoms of patients with FM (14). According to Weinstein et al. (28), patients with se-

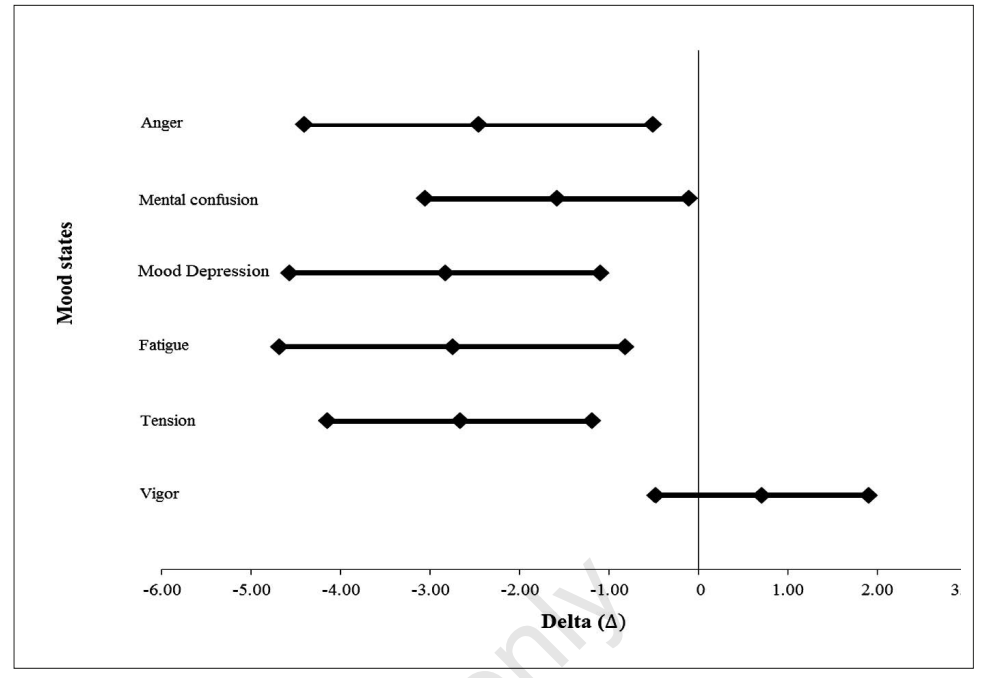

Figure 3 - Mood States after three session of Strength training.

vere depression who undertook a session of physical exercise had a less depressed mood shortly after the session; after 30 minutes, the depressed mood returned to baseline, demonstrating the need for regular physical exercise. In a study carried out by Meyer et al. (39), 24 patients with depression underwent 30 minutes of exercise on a cycle ergometer and presented improvements in mood. A study of multiple sclerosis patients undergoing walking and yoga interventions demonstrated that a few exercise sessions could improve the mood of these patients, significantly influencing feelings of anger, confusion, mood depression and tension (22). This result resembles the findings of the present study, although the former involved different interventions. Our study has some limitations, such as the absence of a control group. However, given the paucity of studies evaluating the acute effect of psychological variables on patients with FM, the results are relevant and present a new perspective on the subject. Further studies and larger samples are required to produce more generalized results. Despite the limitations of this study, it is important to emphasize that the results have immediate clinical implications for the treatment of patients with FM. Improvements in mood states are associated with greater adherence to exercise (40). Given that an improved mood can help 
to relieve FM symptoms, the TF program may offer a non-pharmacological treatment for patients.

\section{CONCLUSIONS}

Based on the results, we can hypothesize that a single strength training session could improve the mood states of patients with fibromyalgia. Reductions in anger, mental confusion, mood depression, fatigue, and tension were observed. After the intervention, patients were also found to have an increase in vigor. We suggest that controlled studies be carried out in the future to confirm this hypothesis.

\section{REFERENCES}

1. Wolfe F, Clauw DJ, Fitzcharles M-AA, et al. 2016 Revisions to the 2010/2011 fibromyalgia diagnostic criteria. Semin Arthritis Rheum. 2016; 46: 319-29.

2. Consoli G, Marazziti D, Ciapparelli A, et al. The impact of mood, anxiety, and sleep disorders on fibromyalgia. Compr Psychiatry. 2012; 53: 962-7.

3. Andrade A, Steffens R de AK, Vilarino GT, et al. Does volume of physical exercise have an effect on depression in patients with fibromyalgia? J Affect Disord. 2017; 208: 214-7.

4. Andrade A, Vilarino GT, Sieczkowska SM, et al. The relationship between sleep quality and fibromyalgia symptoms. J Health Psychol. 2018; 135910531775161.

5. Brandt R, Fonseca ABP, Oliveira LGA de, et al. Perfil de humor de mulheres com fibromialgia. J Bras Psiquiatr. 2011; 60: 216-20.

6. Cassisi G, Sarzi-Puttini P, Alciati A, et al. Symptoms and signs in fibromyalgia syndrome. Reumatismo. 2008; 60: 15-24.

7. Andrade A, Bevilacqua GG, Coimbra DR, et al. Sleep Quality, Mood and Performance: A Study of Elite Brazilian Volleyball Athletes. J Sport Sci Med 2016; 15: 601-5.

8. Terry PC, Lane AM, Fogarty GJ. Construct validity of the Profile of Mood States - Adolescents for use with adults. Psychol Sport Exerc. 2003; 4: 125-39.

9. Zhang CQ, Si GY, Chung PK, et al. Psychometric properties of the Brunel Mood Scale in Chinese adolescents and adults. J Sports Sci. 2014; 32: 1465-76.

10. Brandt RR, de Liz CM, Crocetta TB, et al. Mental health and associated factors in athletes during the Open Games of Santa Catarina. Rev Bras Med Esporte. 2014; 20: 276-80.
11. Brosseau L, Wells GA, Tugwell P, et al. Ottawa panel evidence-based clinical practice guidelines for aerobic fitness exercises in the managment of fibromyalgia: part 1 . Phys Ther. 2008; 88: 857-71.

12. Brosseau L, Wells G, Tugwell P, et al. Ottawa Panel evidence-based clinical practice guidelines for strengthening exercises in the management of fibromyalgia: part 2. Phys Ther. 2008; 88: 873-86.

13. Kelley GA, Kelley KS, Jones DL. Efficacy and effectiveness of exercise on tender points in adults with fibromyalgia: a meta-analysis of randomized controlled trials. Arthritis. 2011; 2011: 1-10.

14. Busch A, Webber S, Richards R, et al. Resistance exercise training for fibromyalgia. Cochrane Database Syst Rev. 2013; 12.

15. Andrade A, Vilarino GT, Serafim TT, et al. Modulation of autonomic function by physical exercise in patients with fibromyalgia syndrome: a systematic review. Pm\&R. 2019 [in press].

16. Kingsley JD, McMillan V, Figueroa A. The effects of 12 weeks of resistance exercise training on disease severity and autonomic modulation at rest and after acute leg resistance exercise in women with fibromyalgia. Arch Phys Med Rehabil. 2010; 91: 1551-7.

17. Andrade A, Steffens R, Sieczkowska S, et al. A systematic review of the effects of strength training in patients with fibromyalgia: clinical outcomes and design considerations. Adv Rheumatol. 2018; 58: 36.

18. Andrade A, Sieczkowska SM, Vilarino GT. Resistance training improves quality of life and associated factors in patients with fibromyalgia syndrome. Pm\&R. 2019; 1-7.

19. Gavi MBROM, Vassalo DV, Amaral FT, et al. Strengthening exercises improve symptoms and quality of life but do not change autonomic modulation in fibromyalgia: a randomized clinical trial. PLoS One. 2014; 9: 8.

20. Ericsson A, Palstam A, Larsson A, et al. Resistance exercise improves physical fatigue in women with fibromyalgia: a randomized controlled trial. Arthritis Res Ther. 2016; 18: 12.

21. Ensari I, Greenlee TA, Motl RW, Petruzzello SJ. Meta-analysis of acute exercise effects on state anxiety: an update of randomized controlled trials over the past 25 years. Depress Anxiety [Internet]. 2015; 32: 624-34.

22. Ensari I, Sandroff BM, Motl RW. Effects of single bouts of walking exercise and yoga on acute mood symptoms in people with multiple sclerosis. Int J MS Care. 2016; 18: 1-8.

23. Slade SC, Dionne CE, Underwood M, Buchbinder R. Consensus on exercise reporting template (CERT): explanation and elaboration statement. Br J Sports Med. 2016; 50: 1428-37. 
24. Herring MP, Hallgren M, Campbell MJ. Acute exercise effects on worry, state anxiety, and feelings of energy and fatigue among young women with probable generalized anxiety disorder: a pilot study. Psychol Sport Exerc. 2017; 33: 31-6.

25. Wolfe F, Smythe HA, Yunus MB, et al. The American College of Rheumatology 1990 criteria for the classification of fibromyalgia. Arthrit Rheum. 1990; 33: 160-72.

26. Rohlfs ICP de M, Rotta TM, Luft CDB, et al. A Escala de Humor de Brunel (Brums): instrumento para detecção precoce da síndrome do excesso de treinamento. Rev Bras Med Esporte. 2008 ; 176-81.

27. Faul F, Erdfelder E, Lang AG, Buchner A. G*Power 3: A flexible statistical power analysis program for the social, behavioral, and biomedical sciences. Behav Res Methods. 2007; 39: 175-91.

28. Weinstein AA, Deuster PA, Francis JL, et al. The role of depression in short-term mood and fatigue responses to acute exercise. Int $\mathrm{J} \mathrm{Be}$ hav Med. 2010; 17: 51-7.

29. Penedo FJ, Dahn JR. Exercise and well-being: a review of mental and physical health benefits associated with physical activity. Curr Opin Psychiatry. 2005; 18: 189-93.

30. Klaperski S, von Dawans B, Heinrichs M, Fuchs R. Does the level of physical exercise affect physiological and psychological responses to psychosocial stress in women? Psychol Sport Exerc. 2013; 14: 266-74.

31. Rebar AL, Stanton R, Geard D, et al. A metameta-analysis of the effect of physical activity on depression and anxiety in non-clinical adult populations. Health Psychol Rev. 2015; 9: 366-78.

32. Byun KH, Hyodo K, Suwabe K, et al. Posi- tive effect of acute mild exercise on executive function via arousal-related prefrontal activations: An fNIRS study. Neuroimage. 2014; 98 : 336-45.

33. Goldenberg DL. The interface of pain and mood disturbances in the rheumatic diseases. Semin Arthritis Rheum. 2010; 40: 15-31.

34. Borchers AT, Gershwin ME. Fibromyalgia: a critical and comprehensive review. Clin Rev Allergy Immunol. 2015; 49: 100-51.

35. Thieme K, Turk DC, Flor H. Comorbid depression and anxiety in fibromyalgia syndrome: relationship to somatic and psychosocial variables. Psychosom Med. 2004; 66: 837-44.

36. Finset A, Wigers SH, Gotestam KG. Depressed mood impedes pain treatment response in patients with fibromyalgia. J Rheumatol. 2004; 31: $976-80$.

37. Bircan C, Karasel SA, Akgun B, et al. Effects of muscle strengthening versus aerobic exercise program in fibromyalgia. Rheumatol Int. 2008; 28: 527-32.

38. Hakkinen A, Hakkinen K, Hannonen P, Alen M. Strength training induced adaptations in neuromuscular function of premenopausal women with fibromyalgia: comparison with healthy women. Ann Rheum Dis. 2001; 60: 21-6.

39. Meyer JD, Koltyn KF, Stegner AJ, et al. Influence of exercise intensity for improving depressed mood in depression: a dose-response study. Behav Ther. 2016; 47: 527-37.

40. Nicolson PJA, Hinman RS, French SD, et al. Improving adherence to exercise: Do people with knee osteoarthritis and physical therapists agree on the behavioural approaches likely to succeed? Arthritis Care Res (Hoboken). 2017; 70: 388-97. 